\title{
Nerve Fiber Growth in Culture on Tissue Substrata from Central and Peripheral Nervous Systems
}

\author{
Salvatore Carbonetto, David Evans, and Philippe Cochard \\ The Neurosciences Unit, Montreal General Hospital Research Institute, McGill University, Montreal, Quebec H3G 1A4, \\ Canada
}

In adult mammals, injured neurons regenerate extensively within the PNS but poorly, if at all, within the CNS. We have studied the effect of substrata consisting of tissue sections from various nervous systems on nerve fiber growth in culture and correlated our results with the growth potential of these tissues in vivo. Ganglionic explants from embryonic chicks (9-12 d) fail to extend nerve fibers onto sections of adult rat optic nerve or spinal cord (CNS) but do so on sciatic nerve (PNS). Dissociated DRG neurons behave similarly whether in serum-containing or defined medium. Tissue substrata from nervous systems that support regeneration in vivo-i.e., goldfish optic nerve, embryonic rat spinal cord, degenerating sciatic nerve-also support fiber growth in culture. Within the same culture, neurons will grow onto sciatic nerve rather than neighboring optic nerve sections, suggesting that the responsible agent(s) is not soluble. In addition, neurons adhere more extensively to sciatic nerve substrata than to optic nerve. The occurrence of 3 molecules known to be involved in neuron-substratum adhesion and nerve fiber growth in vitro has been documented immunocytochemically in the tissue sections. One of these, laminin, is demonstrable in all tissues tested that supported nerve fiber growth. Immunoreactivities for fibronectin and heparan sulfate proteoglycan are found in only some of these tissues. None of these 3 molecules can be demonstrated in neural cells of normal adult rat CNS tissue. Our data suggest that these molecules may be important effectors of nerve regeneration in neural tissues.

Following trauma to the CNS of adult mammals, nerve fiber growth is meager and functional recovery correspondingly poor. In contrast, neurons of the PNS may extend nerve fibers for many centimeters, reconnecting more or less accurately with their targets. Aguayo and coworkers have shown that some axotomized CNS neurons have the capacity to regenerate their axons for considerable distances into peripheral nerve grafts (Richardson et al., 1980; David and Aguayo, 1981; Aguayo, 1985). Moreover, neither PNS (Nathaniel and Pease, 1963; Nathaniel and Nathaniel, 1973; Aguayo et al., 1979) nor CNS

\footnotetext{
Received Mar. 31, 1986; revised Aug. 6, 1986; accepted Aug. 11, 1986.

We thank Dr. John Hassell, NIH, for supplying antibodies to heparan sulfate proteoglycan. We also thank Dr. R. Levine for his critique of the manuscript. D.E. was a McGill University summer research fellow and P.C. the recipient of NATO and CNRS postdoctoral fellowships. This work was supported by grants to S.C. from NIH (NS19068) and MRC (MA-9000).

Correspondence should be addressed to Dr. S. Carbonetto, Montreal General Hospital Research Institute, McGill University, 1650 Cedar Avenue, Montreal, P.Q. H3G 1A4, Canada.

Copyright (C) 1987 Society for Neuroscience $0270-6474 / 87 / 020610-11 \$ 02.00 / 0$
}

neurons are able to clongatc into the adult CNS. Taken together, these data suggest that heterogeneity in the microenvironment of CNS and PNS neurons is largely responsible for their disparate response to axotomy.

Hypotheses regarding the molecular basis of axonal regeneration are often grounded in observations made on nerve fibers growing in culture. Cultured neurons require 2 major environmental influences to elaborate nerve fibers, growth factors (reviewed by Berg, 1984) and a properly adhesive substratum (Letourneau, 1975; Greene and Tischler, 1976). NGF, the bestknown of the growth factors, is required for the survival and growth of dorsal root ganglion (DRG) and sympathetic neurons in vivo as well as in culture (reviewed by Greene and Shooter, 1980). Other growth factors have been identified (Collins, 1978; Barde et al., 1982), but their function in vivo has not been described.

Several adhesive molecules mediate the attachment of ncurons to culture substrata in addition to supporting nerve fiber growth (Letourneau, 1975; Akers et al., 1981; Baron van Evercooren et al., 1982; Carbonetto et al., 1982; Manthorpe et al., 1983; Rogers et al., 1983; Rauvala, 1984; Smalheiser et al., 1984). Three of these, the adhesive proteins laminin, fibronectin, and collagen, are components of the extracellular matrix of the nervous system and may function in vivo as effectors of nerve fiber growth. For fibronectin (Carbonetto et al., 1983) and laminin (Edgar et al., 1984) growing nerve fibers interact with a discrete region of the protein, as might occur during ligand receptor binding. Interestingly, laminin alone can mimic some of the trophic properties of NGF (Edgar et al., 1984; Davis et al., 1985; Lander et al., 1985), suggesting that this large multifunctional molecule has domain(s) that are neurotrophic and/ or adhesive for cells. The function of these adhesive proteins during nerve regeneration in vivo has not been tested.

This paper describes our attempts to analyze some of the environmental influences within the nervous system affecting nerve fiber growth. In our studies, neurons were cultured on sections of neural tissues from PNS or CNS. The neurons failed to extend nerve fibers on tissue substrata from adult rat CNS (optic nerve, spinal cord), whereas extensive nerve fiber growth occurred on tissue from PNS (sciatic nerve). In addition, goldfish optic nerve and embryonic rat spinal cord, which support nerve fiber growth in vivo, did so in our culture system. The difference in the growth of neurons on these tissue substrata appears to reflect the inactivity or absence of adhesive molecules in the adult mammalian CNS tissues. Several extracellular matrix (ECM) components, mentioned above (laminin, fibronectin, heparan sulfate proteoglycan), that are demonstrable immunocytochemically in the PNS are essentially absent in the adult CNS. 

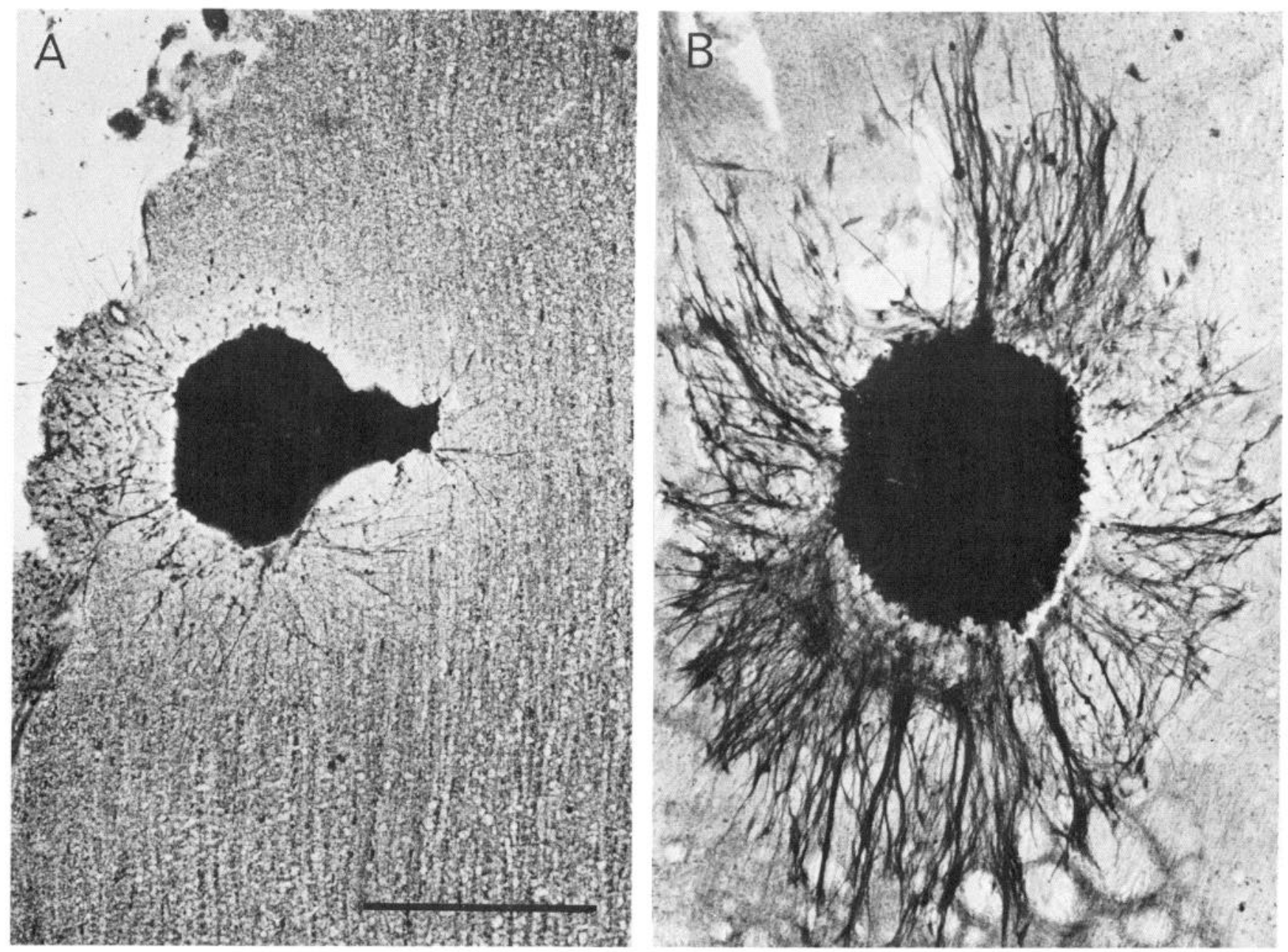

Figure 1. Growth of DRG explants on substrata from spinal cord and sciatic nerve. $A$, Ganglia attached to sections of CNS tissue generally did not grow but occasionally extended several short processes such as the "minimal" growth shown here. Calibration bar, $0.5 \mathrm{~mm}$. $B$, By comparison, ganglia attached to sciatic nerve sections grew extensively (same magnification as in $A$ ).

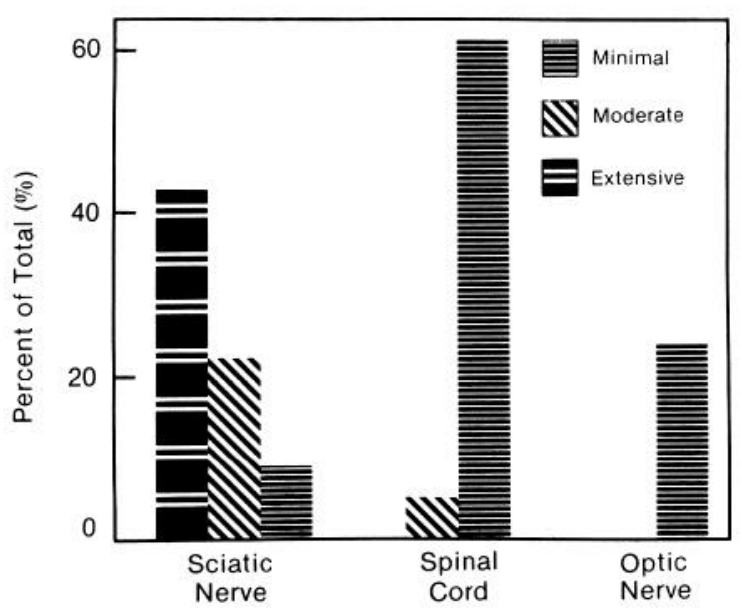

Figure 2. Nerve fiber growth by DRG explants on CNS and PNS tissue substrata. Chick DRGs were seeded onto substrata as in Figure 1. Only ganglia directly attached to tissue sections were included in this analysis. The ordinate shows the percentage of those ganglia evaluated that grew minimally, moderately, or extensively (see Materials and Methods). Number of ganglia evaluated: sciatic nerve, $n=86$; spinal cord, $n=$ 18 ; optic nerve, $n=63$.
Our data suggest that adhesive molecules within the ECM may be important determinants of nerve fiber growth in culture and in vivo.

\section{Materials and Methods}

Preparation of tissue substrata. Adult and embryonic Sprague-Dawley rats (Charles River), fertilized chicken eggs, and mature goldfish (supplied by Dr. R. Levine, McGill University) were used throughout this study. Adult rats were sacrificed by an overdose of chloral hydrate and perfused through the heart with PBS $(0.1 \mathrm{~m}, \mathrm{pH} 7.2)$. Segments of optic nerve, spinal cord, and sciatic nerve were excised. In all cases, sciatic nerves were desheathed and optic nerves and spinal cord freed of meninges. In experiments with injured PNS and CNS tissues, substrata were prepared from rats that had optic and sciatic nerve transections performed unilaterally under chloral hydrate anesthesia. Degenerating nerves were excised and used 5-10 d later. The tissue was then mounted, frozen in liquid nitrogen, and either stored at $-70^{\circ} \mathrm{C}$ or cut into sections on a cryomicrotome. Sections were mounted onto round coverslips $(15 \mathrm{~mm}$ in diameter) of glass or tissue culture plastic (Thermanox, Lux) that had been coated overnight with polylysine $(1 \mathrm{mg} / \mathrm{ml}$ in borate buffer, $\mathrm{pH}$ 9). Prior to use, these substrata were sterilized by brief uv irradiation and washed extensively in culture medium.

Preparation of neuronal cultures. For most of our experiments, cultures of ganglionic explants from DRG or sympathetic ganglia were prepared from 9-12 d chick embryos by conventional methods (Carbonetto and Fambrough, 1978). Cells were cultured either in Dulbecco's Minimum Essential Medium (DMEM) plus 10\% fetal calf serum, cy- 

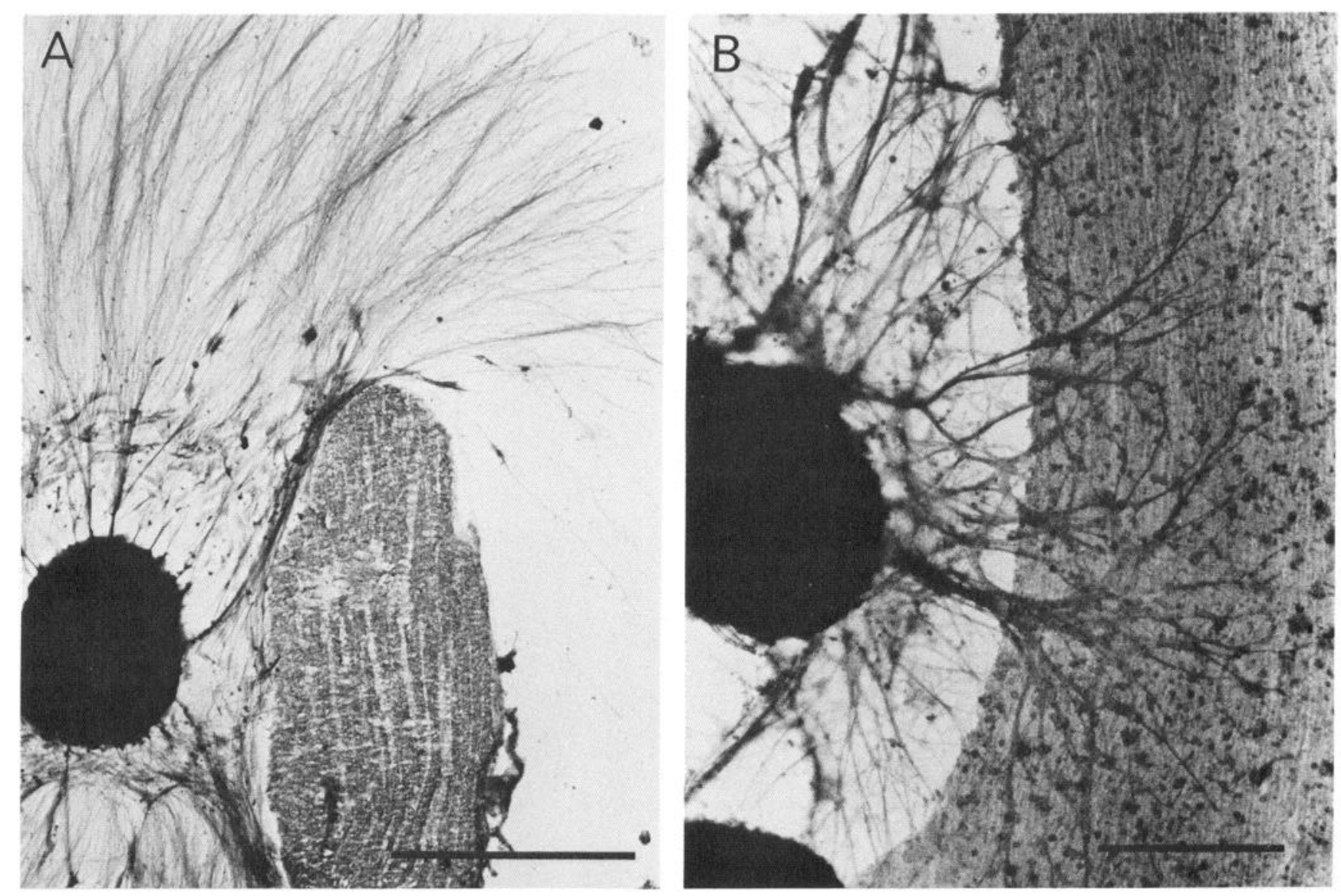

Figure 3. Nerve fiber growth onto sciatic nerve and optic nerve tissue from distant ganglia. Ganglia generally grew extensively on the polylysinecoated plastic, producing a halo of fine nerve fibers that by $2 \mathrm{~d}$ in culture were essentially free of non-neuronal cells. $A$, These fibers approached a section of optic nerve and grew along the periphery of the section but did not grow onto the section. Calibration bar, $0.5 \mathrm{~mm}$. $B$, Ganglia distant from sections of sciatic nerve grew extensively on the culture plastic and onto the tissue substrata. Calibration bar, $0.25 \mathrm{~mm}$.

tosine arabinoside $(20 \mu \mathrm{M})$, and gentamicin or in defined medium (Bottenstein and Sato, 1978). Unless noted, all media contained NGF (50 $\mathrm{ng} / \mathrm{ml})$. Cultures of dissociated cells enriched for neurons $(>80 \%$ neurons) were prepared by briefly treating ganglia with trypsin, triturating them, and plating the dissociated cells on petri dishes coated with serum. During this preplating step non-neuronal cells (fibroblasts and glia) preferentially adhere to the culture substratum and the cells enriched for neurons can be harvested by simply shaking the dish and aspirating the medium. PC12 cells were maintained as described by Greene and Tischler (1976) and treated with NGF for approximately $6 \mathrm{~d}$ before seeding onto tissue substrata. Ganglionic explants or dissociated cells $\left(2 \times 10^{5}\right.$ cells $/ \mathrm{ml}$ ) were seeded onto coverslips coated with tissue sections $(100$ $200 \mu \mathrm{l} /$ coverslip) placed in $35 \mathrm{~mm}$ culture dishes. Cultures were fed the following day with fresh medium and maintained for 2-5 d to allow nerve fiber growth.

Quantification of neuron-substratum adhesion and nerve fiber growth. Neuronal somata or nerve fibers from ganglionic explants located directly on tissue sections could not be reliably visualized in living cultures. To quantify neuronal adhesion and nerve fiber growth, the cultures were washed with DMEM, fixed with paraformaldehyde $(4 \%$ in $0.1 \mathrm{M}$ phosphate buffer, $\mathrm{pH}$ 7.2), rinsed in PBS, and stained with Coomassie Blue $(0.1 \%$ in $10 \%$ acetic acid, $25 \%$ propanol) for $4 \mathrm{~min}$. Following staining, nerve fibers were easily visible on tissue sections, as were any non-neuronal cells.

Neuron substratum adhesion was quantified by seeding DRG cells enriched for neurons and counting the somata that attached to tissue sections. The cell density was determined by counting the total number of cells attached per substratum and measuring the surface area of the tissue substrata with an IBAS image analyzer (Zeiss).

Nerve fiber growth from ganglionic explants was separated into 2 categories: (1) growth from ganglia attached to and growing directly on top of tissue substrata or (2) growth from ganglia attached to the culture plastic at some distance from the tissue and subsequently growing onto it. In the first category, nerve fibers that grew a distance of about 1 diameter of the ganglion were considered to be growing minimally. Less than this distance was considered negligible growth, and more than this was moderate (approximately 2 diameters of the ganglion) or extensive

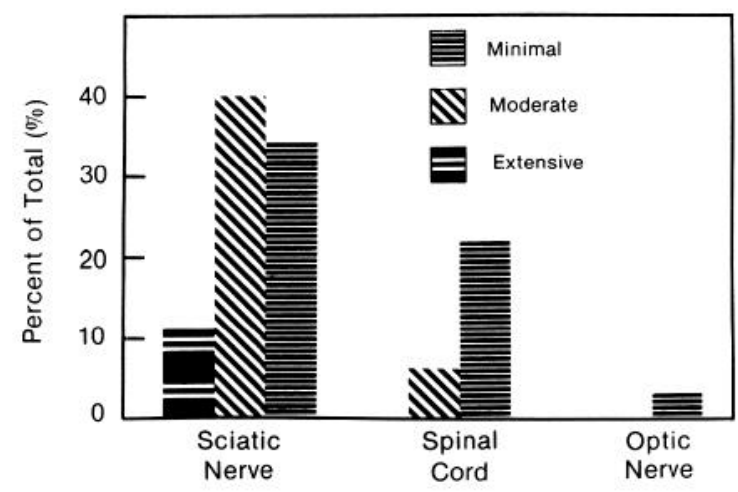

Figure 4. Nerve fiber growth onto CNS and PNS tissue substrata from DRGs distant from the tissue. Cultures were prepared as in Figure 3. Only ganglia not in contact with tissue sections and clearly growing fibers were included in this analysis. The ordinate gives the percentage of ganglia growing on the culture plastic that grew minimally, moderately, or extensively on the tissue. Number of ganglia: sciatic nerve, $n=$ 65 ; spinal cord, $n=18$; optic nerve, $n=36$. 


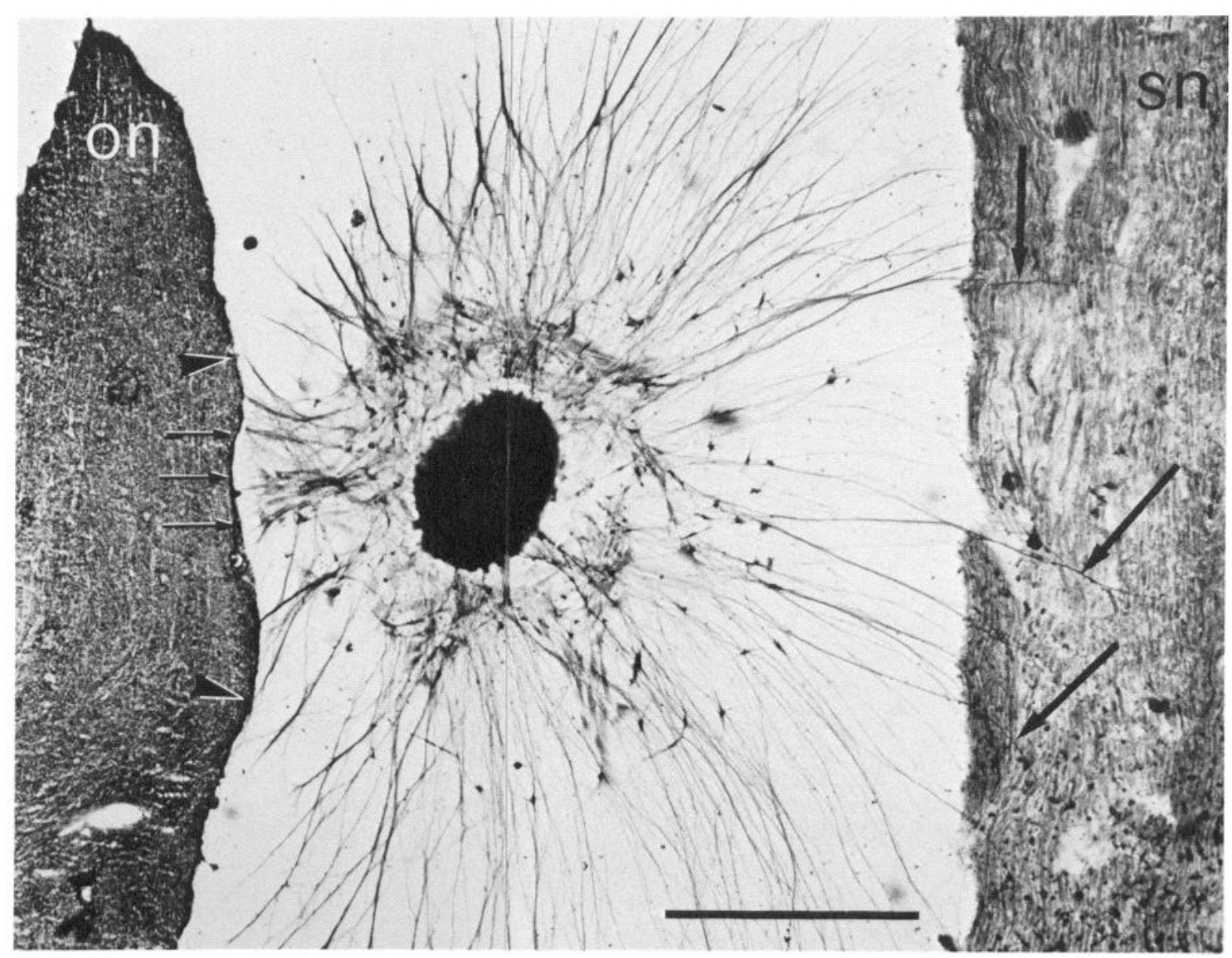

Figure 5. Nerve fiber growth on sciatic nerve and optic nerve sections within the same culture. For these experiments, tissue sections of sciatic nerve and optic nerve were placed side by side and ganglia seeded between the sections. The ganglion grew extensively up to the optic nerve section but not onto it. Fibers can be seen growing around the section (arrowheads), while others may have stopped growing altogether (small arrows). Growth of the same ganglion to the somewhat more distant sciatic nerve section resulted in readily observable growth of the fibers onto the tissue (arrows). Calibration bar, $0.5 \mathrm{~mm}$.

growth (more than 2 diameters of the ganglion). In the second category, growth onto the tissue substrata from distant ganglia was quantified as minimal ( $<10$ fibers on the tissue), moderate (10-50 fibers on the tissue), or extensive ( $>50$ fibers on the tissue). For culture of dissociated neurons, individual neurons and all aggregates extending nerve fibers on the tissue were summed, and neuronal growth was expressed as a percentage of the total number of aggregates attached to the tissue substrata. Data were analyzed for statistical significance by comparing means directly for the least significant difference (Steel and Torrie, 1980).

Immunocytochemistry. For immunocytochemical localization of extracellular matrix components (laminin, fibronectin, heparan sulfate proteoglycan), rats were perfused with $4 \%$ paraformaldehyde and the tissues of interest excised. The tissue was sectioned $(10 \mu \mathrm{m})$ on a cryostat as in preparing tissue substrata. Sections were then preincubated for 30 min in 3\% albumin in PBS and subsequently incubated overnight with affinity-purified antibodies to horse serum fibronectin, and antisera to EHS laminin and to heparan sulfate proteoglycan (a gift from Dr. John Hassel, NIH). The antibodies were thoroughly washed free from the tissue, the sections incubated with FITC-conjugated goat anti-rabbit IgG (Cappel), thoroughly washed again in PBS, and mounted in Trisbuffered glycerol ( $\mathrm{pH} 9)$.

\section{Results}

Nerve fiber growth by ganglionic neurons on adult rat sciatic nerve, optic nerve, and spinal cord

Explants of DRG or sympathetic ganglia attached to substrata of sciatic nerve, optic nerve, or spinal cord from adult rats. Once attached to sciatic nerve sections, the ganglia extended a halo of nerve fibers onto the tissue and surrounding culture plastic (Figs. 1B,2). In contrast, ganglia attached to optic nerve or spinal cord substrata grew poorly, if at all, on the CNS tissue (Fig. $1 A)$. For example, $66 \%(n=68)$ of the ganglia on sciatic nerve grew moderately compared with none on optic nerve ( $n=63$ ) (Fig. 2).

Ganglionic explants contain non-neuronal cells (Schwann cells and fibroblasts) as well as neurons. These non-neuronal cells are known to stimulate nerve fiber outgrowth from neurons attached to them (Ludueña, 1973), as well as from neurons placed on substrata coated with materials they produce (Helfand et al., 1976; Collins, 1978; Hawrot, 1980). This raises the possibility that the tissue sections are acting indirectly, through ganglionic non-neuronal cells, to affect nerve fiber growth. To examine this possibility we analyzed the growth of ganglionic explants attached to the culture plastic distant from the tissue sections. In these experiments, cytosine arabinoside in the medium inhibited cell division and the halo of nerve fibers extending from ganglia was largely free of non-neuronal cells (Fig. 3) (Wood, 1976). Of the ganglia growing to contact sciatic nerve, more than $72 \%(n=65)$ grew at least minimally onto the tissue substratum (Figs. 3B, 4). In comparison, $98 \%$ of the ganglia $(n=36)$ that had fibers contacting sections of optic nerve and $78 \%$ of those contacting spinal cord $(n=18)$ showed no growth onto the tissue. In several instances nerve fibers were found to 

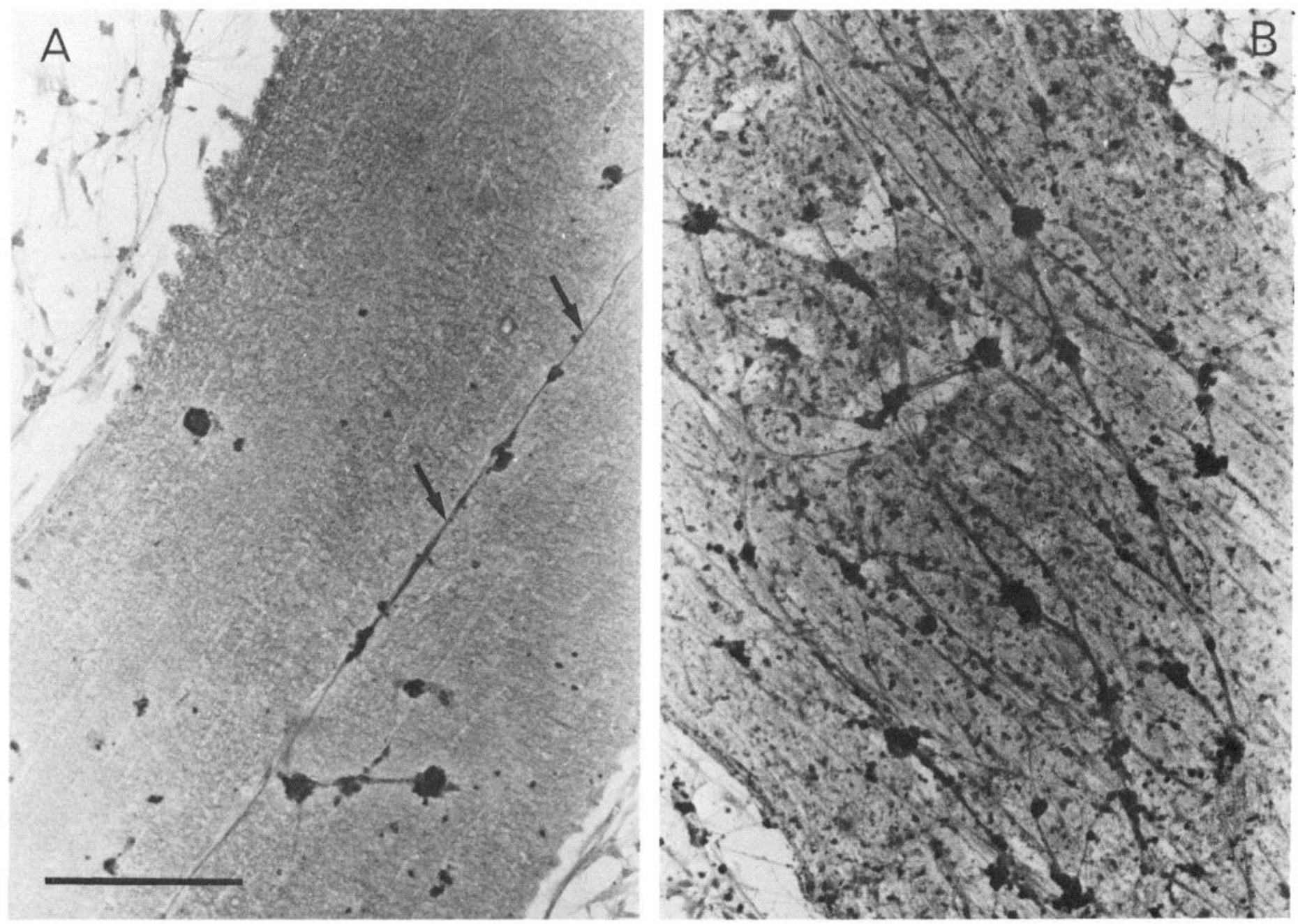

Figure 6. Dissociated DRG neurons on sections of optic nerve and sciatic nerve. $A$, Sections of optic nerve had few cells attached, and these showed little fiber growth. Interestingly, there was frequent attachment and fiber growth along the periphery of the tissue, which, in this culture, appears as a "septum" between 2 adjacent sections (arrows). Calibration bar, $250 \mu \mathrm{m}$. B, On sections of sciatic nerve, dissociated cells attached, and extended nerve fibers readily. Same magnification as in $A$.

grow on the periphery of the CNS tissue (Fig. $3 A$ ), as if growing on the residual connective tissue sheath. Culturing DRG cells in serum-free medium (Bottenstein and Sato, 1978) yielded the same results: growth on PNS (sciatic nerve) but not on CNS (optic nerve) substrata. Thus, neither non-neuronal cells or their products nor serum components such as fibronectin appear to modify tissue substrata such that growth results only on PNS substrata.

The pattern of nerve fiber growth on the culture coverslip but not on the optic nerve substratum nearby suggests a local effect rather than one produced by factors diffusing some distance from the tissue. For example, outgrowth from ganglionic areas adjacent to sciatic nerve sections was not obviously more extensive than from regions of the ganglia away from the section. Similarly, fibers from ganglia near optic nerve grew to contact the tissue before growing around it (Fig. $3 A$ ). To determine more directly whether a diffusible agent(s) might be responsible for the growth of DRG explants on sciatic nerve but not CNS, ganglia were cultured on coverslips between sections of optic or sciatic nerve. Among those that grew to contact both PNS and CNS substrata $(n=23), 70 \%$ ( 8 minimal growth, 8 moderate growth) showed a marked preference for sciatic nerve (Fig. 5). In no case was there any growth on optic nerve. In a smaller sample where ganglia were given a "choice" of spinal cord or sciatic nerve, $75 \%$ of the cases showed growth on sciatic nerve with none on spinal cord $(n=4)$.

The growth of these distant ganglia whose nerve fibers are free of non-neuronal cells onto sciatic but not optic nerve is consistent with the hypothesis that the fibers are responding to the tissue sections themselves and not to factors produced by non-neuronal cells (see Discussion). Moreover, the pattern of outgrowth of ganglia placed between optic and sciatic tissues suggests that this effect is not mediated by a diffusible substance(s) but by direct neuron-substratum contact.

\section{Dissociated neurons: adhesion to and growth on sciatic nerve and optic nerve substrata}

Ganglionic explants are covered by a capsule of connective tissue that is difficult to remove and probably restricts outgrowth from regions of ganglia or inhibits growth entirely (approximately $10-25 \%$ of our ganglionic explants failed to extend processes on any substratum). By preparing enriched cultures of DRG and sympathetic neurons it was possible to avoid the limitations resulting from the encapsulating connective tissue and the variability of fiber outgrowth from ganglia. Dissociated DRG neurons behaved qualitatively the same as explanted gan- 


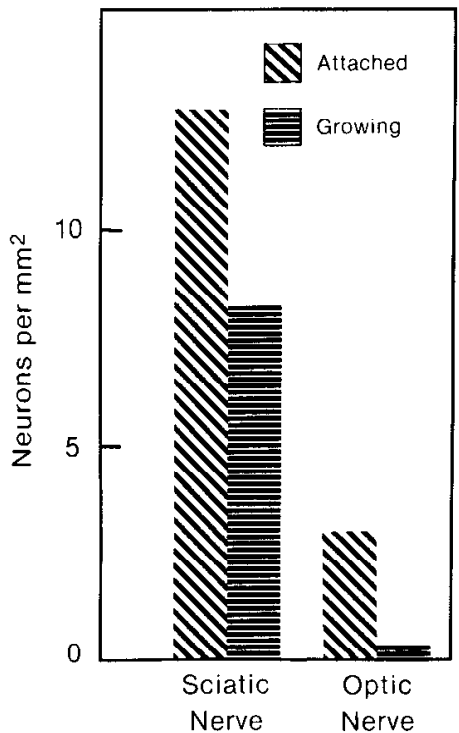

Figure 7. Quantification of attachment and growth of dissociated DRG neurons on sections of optic and sciatic nerves. The ordinate gives the mean density of cells and cell-aggregates attached to the sections and extending nerve fibers onto them.

glia, growing on sciatic nerve substrata but not on optic nerve. Although seeded as single cells, the neurons often aggregated into small clumps on the tissue substrata (Fig. 6). By $2 \mathrm{~d}$ in culture there were fewer cells attached to optic nerve substrata than to sciatic nerve (Figs. 6,7), and the outgrowth of nerve fibers was much greater on sciatic nerve than on optic nerve ( 8.7 aggregates $/ \mathrm{mm}^{2}$ on sciatic nerve vs 0.4 aggregates $/ \mathrm{mm}^{2}$ on optic nerve). Often the pattern of growth in these cultures appeared to follow the longitudinal axis of the sciatic nerve (Fig. $6 B)$.

The reduced number of cells attached to the optic nerve sections could have arisen either from diminished adhesion or enhanced detachment of neurons from optic nerve. Cultured cells are well known to adhere selectively to culture substrata and other cells. Neurons in culture will also detach selectively from other cells (Ruffolo et al., 1978). The kinetics of attachment of DRG cells to sciatic nerve and optic nerve substrata (Fig. 8) indicated that even by $1 \mathrm{hr}$ attachment to sciatic nerve was significantly greater than to optic nerve and this attachment increased monotonically over the next $4 \mathrm{hr}$. There was no evidence for selective detachment of cells from optic nerve that could be responsible for the 7-fold difference between the number of cells attached to sciatic nerve and to optic nerve.

\section{Other sources of neurons and tissue substrata}

It is well known that culture plastic coated with basic polyaminoacids such as polylysine or polyornithine facilitate neuronsubstratum adhesion (Fig. 3) (Letourneau, 1975). However, polylysine would not be expected to serve as a substratum for nerve fiber growth in vivo since it is not likely to be present on the surfaces of neural cells. Nevertheless, the robust effects of polylysine-coated substrata in supporting nerve fiber growth caution that molecules only partially related or entirely distinct from those operating in vivo may stimulate fiber growth in culture. With this in mind, we examined the effects of CNS and PNS tissues from a variety of sources to correlate their known efficacy at supporting nerve fiber growth in vivo with that in

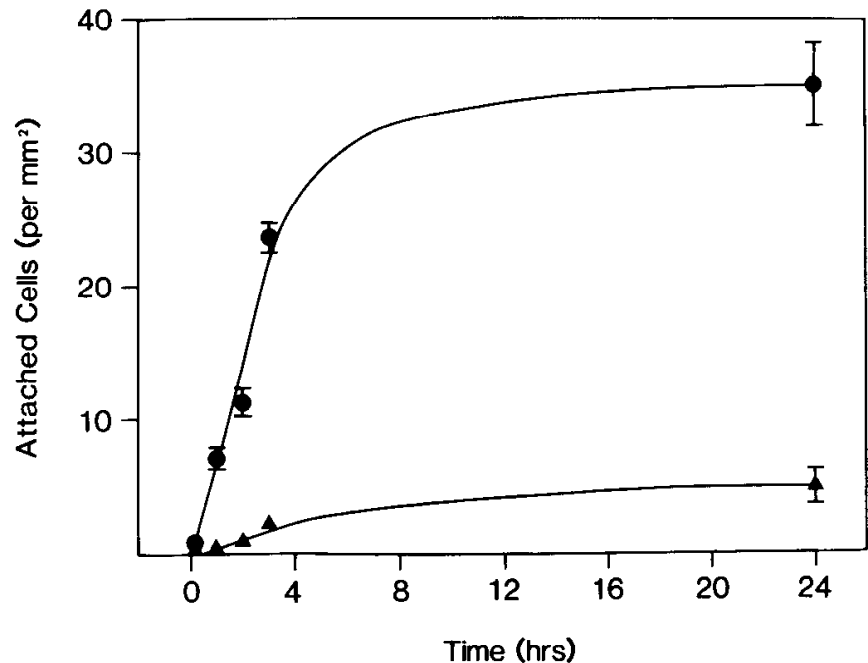

Figure 8. Kinetics of DRG cell attachment to substrata of optic nerve and sciatic nerve. DRG cells enriched for neurons were seeded onto substrata of sciatic nerve ( $(\boldsymbol{\theta})$ or optic nerve $(\boldsymbol{\Delta})$. At times indicated along the abscissa cultures were removed from the incubator, washed gently with HBSS, and fixed. The number of attached cells was quantified by light-microscopic observation (see Materials and Methods). The error bars are shown where they are larger than the symbol and represent the SEM. The number of attached cells counted ranged from $8 /$ culture (optic nerve at $10 \mathrm{~min}$ ) to $629 /$ culture (sciatic nerve at $24 \mathrm{hr}$ ).

culture. Like adult optic nerve, spinal cord from adult rats supported dramatically less nerve fiber growth from dissociated neurons than did sciatic nerve (Figs. 9D, 10), thus confirming results obtained with whole ganglia (see Fig. $1 A$ ). However, substrata of spinal cord tissue from 14 and $16 \mathrm{~d}$ embryonic rats supported extensive fiber growth in culture, approaching that of sciatic nerve (Figs. $9 C, 10$ ). On substrata of optic nerve from mature goldfish substantially less nerve fiber growth was observed than on either sciatic nerve or embryonic spinal cord sections but there was significantly more than on adult rat spinal cord (Figs. 9B, 10). Substrata of degenerating rat sciatic nerve (Fig. 9A) were also effective for fiber growth but not obviously more so than normal sciatic nerve (Fig. $6 B$ ). In conclusion, several substrata from nervous tissue (sciatic nerve, embryonic spinal cord, goldfish optic nerve) that support nerve fiber growth in vivo also support significant growth of DRG neurons in culture. Other substrata (adult rat optic nerve and spinal cord) that support little fiber growth in vivo support correspondingly little growth in culture. This correlation of activity in vivo with that in culture supports the notion that molcculcs residing in tissue substrata contribute to the control of nerve fiber growth in vivo. Moreover, the growth of neurons on rat sciatic nerve but not on optic is not restricted to DRG neurons. Similar results were found with explants of embryonic chick sympathetic ganglia, dissociated sympathetic neurons, and $\mathrm{PC} 12$ cells (data not shown).

\section{Immunocytochemical localization of extracellular matrix components in PNS and CNS substrata}

Regenerating nerve fibers in vivo are often found in close apposition to basement membranes (Ide et al., 1983). Among the molecular constituents of basement membranes, fibronectin (Akers et al., 1981; Carbonetto et al., 1982; Rogers et al., 1983), Type IV collagen (Carbonetto et al., 1983), laminin (Baron van Evercooren et al., 1982; Manthorpe et al., 1983; Rogers et al., 

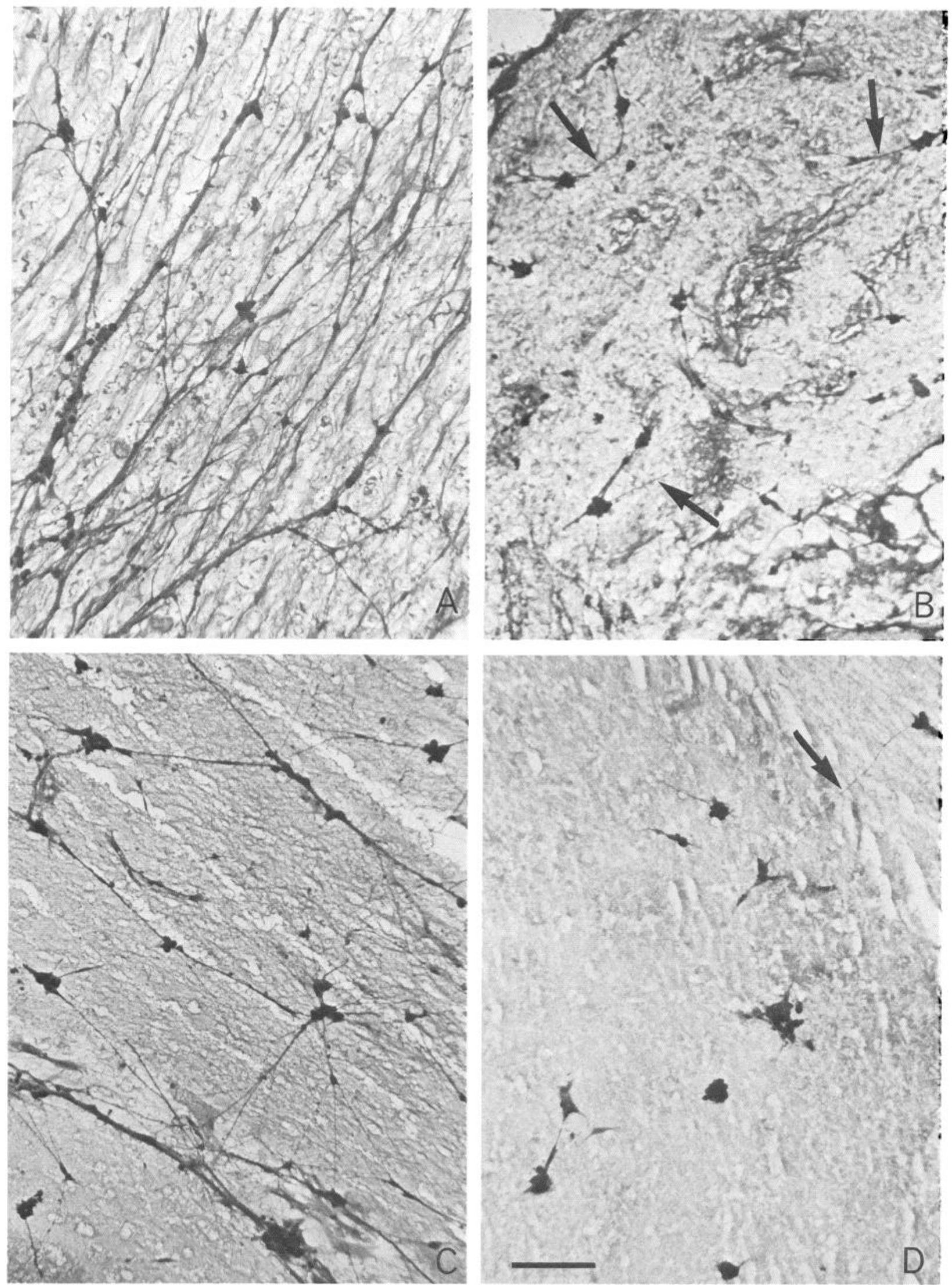

Figure 9. Nerve fiber growth on various neural tissue substrata. $A$, Extensive nerve fiber outgrowth occurred on sections of degenerating rat sciatic nerve, as it did on normal nerve (see Fig. $6 B$ ). Note that in both cases the general pattern of nerve fiber outgrowth followed the long axis of the nerve. $B$, Nerve fiber outgrowth (arrows) was also observed on sections of goldfish optic nerve, although it was much less extensive than on rat PNS tissue. $C$, Dissociated DRG neurons also readily extended long, thin processes on sections of embryonic ( $16 \mathrm{~d})$ rat spinal cord. $D$, In comparison, fiber outgrowth was virtually absent on adult rat spinal cord, except in rare instances (arrow). Scale bar in $D$ represents $100 \mu \mathrm{m}$ and applies to all micrographs. 


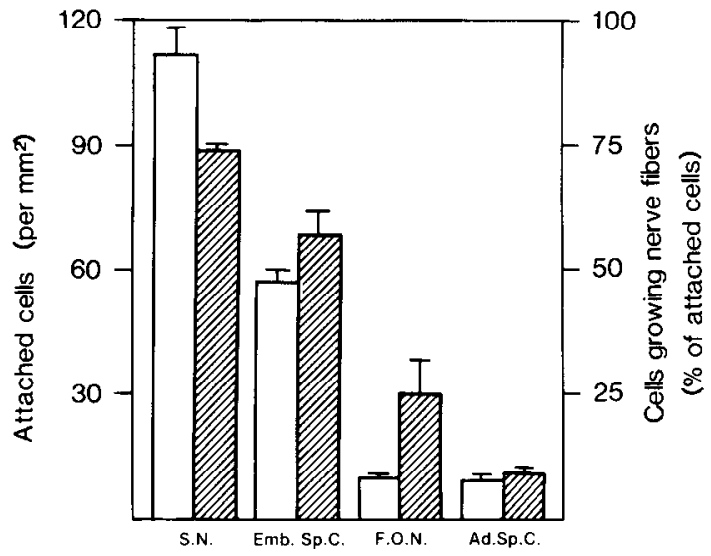

Figure 10. Quantification of fiber growth on various neural tissue substrata. Dissociated DRG neurons were seeded onto substrata (3 cultures each) of the following tissues: rat sciatic nerve (S.N.), spinal cord from $14 \mathrm{~d}$ embryonic rats (Emb.Sp.C.), goldfish optic nerve (F.O.N.), and aduit rat spinal cord (Ad.Sp.C.). After approximately $2 \mathrm{~d}$ the cultures were fixed and the number of attached cells (open bars, left ordinate) and neurons growing nerve fibers were counted (shaded bars, right ordinate). The numbers of attached cells counted ranged from 220 to 738 / culture. The number of neurons growing nerve fibers counted ranged from 49 to 528 /culture. Sciatic nerve and embryonic spinal cord $(p<$ $0.001)$, as well as fish optic nerve $(p<0.05)$, were significantly better substrata for nerve fiber growth than adult spinal cord sections.

1983; Smalheiser et al., 1984; Lander et al., 1985), and heparan sulfate proteoglycan (Culp et al., 1980) have all been implicated in neuron substratum adhesion and/or nerve fiber growth. Since our studies indicate that neurons adhere less well to CNS than to PNS tissues, we sought to localize immunocytochemically some of these ECM components in our tissue substrata (Fig. 11).

Immunoreactivities for laminin, heparan sulfate proteoglycan, and fibronectin were readily detected in normal and degenerating sciatic nerves. Laminin and heparan sulfate proteoglycan were clearly restricted to a thin layer in the endoneurium, most probably the basement membrane. Fibronectin appeared more diffuse in the endoneurium and perineurium. None of these ECM components could be found within the optic nerve or spinal cord of adult rats, except in the external connective tissue layer and in the basement membranes of blood vessels. Interestingly, in 14 and $16 \mathrm{~d}$ embryonic spinal cord, striking differences were observed in the staining patterns of these various antigens. Laminin was present almost throughout the tissue surrounding neuroepithclial cclls, whercas fibroncetin and heparan sulfate proteoglycan were largely restricted to the connective tissue and vascular components, as in adult CNS tissue. Laminin was also detectable in fish optic nerve, localized in septa partitioning nerve bundles, but more diffusely than in the basal lamina of peripheral nerve. Low but detectable levels of fluorescence for fibronectin and heparan sulfate proteoglycan were also found in punctate and fibrillar distributions in the fish optic nerve. Identical localizations for all these antigens were found in tissue sections used as culture substrata for $2 \mathrm{~d}$, indicating that these molecules persist in culture and are likely available to interact with nerve fibers.

\section{Discussion}

The purpose of our studies was to explore in vitro the properties of neural tissue with regard to its ability to support nerve re- generation. The approach we have taken, using sections of neural tissues as culture substrata, was prompted by 2 sets of experiments. First, Ide and coworkers (1983) reported that freezethawed peripheral nerves grafted into the CNS of adult rats supported some nerve fiber growth. Second, Schwab and Thoenen (1985) described the first system of cultured sciatic and optic nerve wherein axons from cultured neurons grew preferentially into explants of the PNS tissue. Indeed, our work should be viewed as an extension of that of Schwab and Thoenen (1985), as well as that of Sandrock and Matthew (1985), who reported a culture system apparently identical to the one we have described here. This culture system uses sections of neural tissue, rather than organ-cultured optic and sciatic nerves (Schwab and Thoenen, 1985), as substrata for embryonic neurons. This method allows direct visualization of nerve fiber growth on the tissues at the light-microscopic level without resort to light- or electron-microscopic analysis of sectioned material. Substantial nerve fiber growth can be seen in 1-2 d, rather than several weeks, and the fiber growth is easily quantified following fixation and staining with Coomassie Blue (Materials and Methods). In addition, the substrata of tissue sections on glass or plastic coverslips are easy to prepare and neurons can be given a choice of CNS or PNS substrata (Fig. 5) without constructing 3-chamber culture vessels (Campenot, 1977; Schwab and Thoenen, 1985).

Our data are in substantial agreement with those of Schwab and Thoenen (1985) and Sandrock and Matthew (1985). We find that nerve fiber growth is significantly greater on PNS tissue than on adult CNS tissue. Additionally, we show that several neural tissues that support nerve fiber growth in vivo (normal and degenerating rat sciatic nerve, embryonic rat spinal cord, fish optic nerve) also support growth of cultured neurons. Neural tissues that do not support fiber growth in vivo (adult rat optic nerve and spinal cord) also support little growth in culture. The observed difference between CNS and PNS substrata does not appear to be due to a diffusible agent(s) released from the tissue (Schwab and Thoenen, 1985) but rather to a local effect of the tissue. We believe this local effect requires contact of the neurons with the tissue and is manifest in the greater adhesion of DRG cells to sciatic nerve than to optic nerve.

It is important to consider whether neurons interact directly with the tissue substrata and not with other cultured cells, their products, or the constituents of the culture medium. In our studies, ganglionic explants produce halos of axons essentially devoid of non-neuronal cells, which nevertheless grow onto sections of sciatic nerve but not optic nerve. This suggests that sciatic nerve sections do not act through non-neuronal cells to stimulate nerve fiber growth. Furthcrmore, growth of neurons in serum-free medium suggests that fibronectin or other serum components that can mediate fiber growth are not binding to sciatic nerve sections so as to enhance fiber growth. However, we cannot eliminate the possibility that the cells themselves produce some molecules that attach preferentially to sciatic nerve to stimulate growth. Even though we see differential adhesion to sciatic and optic substrata within $1-2 \mathrm{hr}$, the cells might, in that brief period, be modifying the substrata.

The simplest explanation for the growth of nerve fibers on PNS, but not CNS, substrata is that a single, positive effector of growth is present in sciatic nerve but not in optic nerve. Alternatively, there may be a mix of negative (Schwab and Thoenen, 1985) and positive effectors in optic nerve and sciatic nerve, respectively. Sandrock and Matthew (1985) reported that a monoclonal antibody that binds to a heparan sulfate proteo- 


\section{SN}
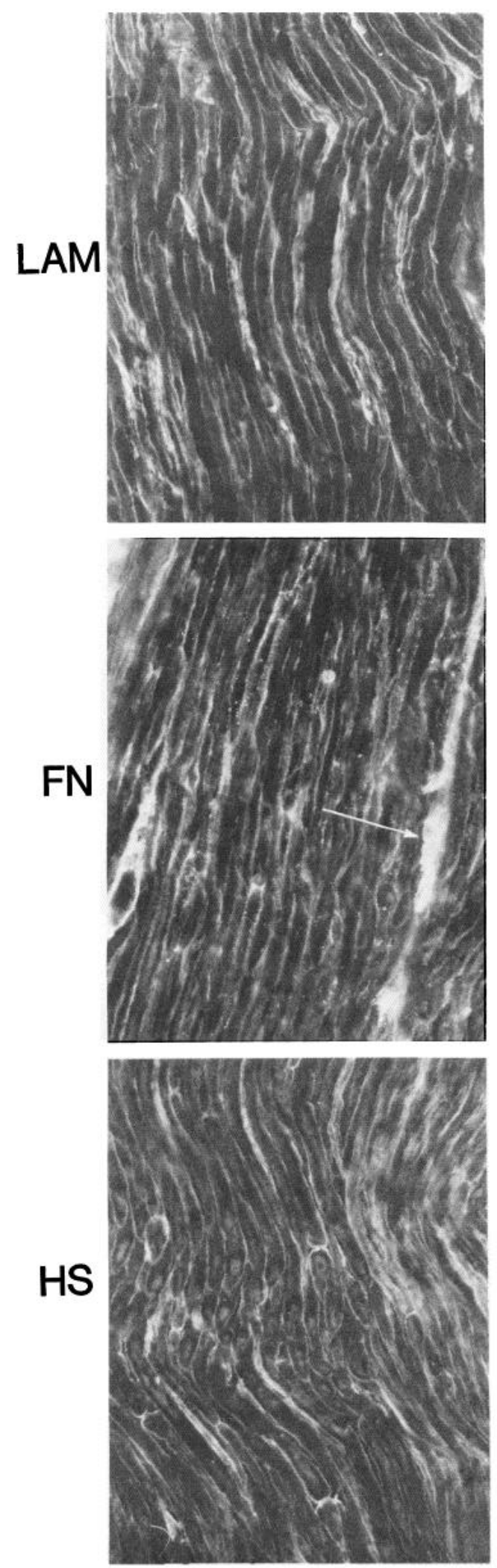

ESC
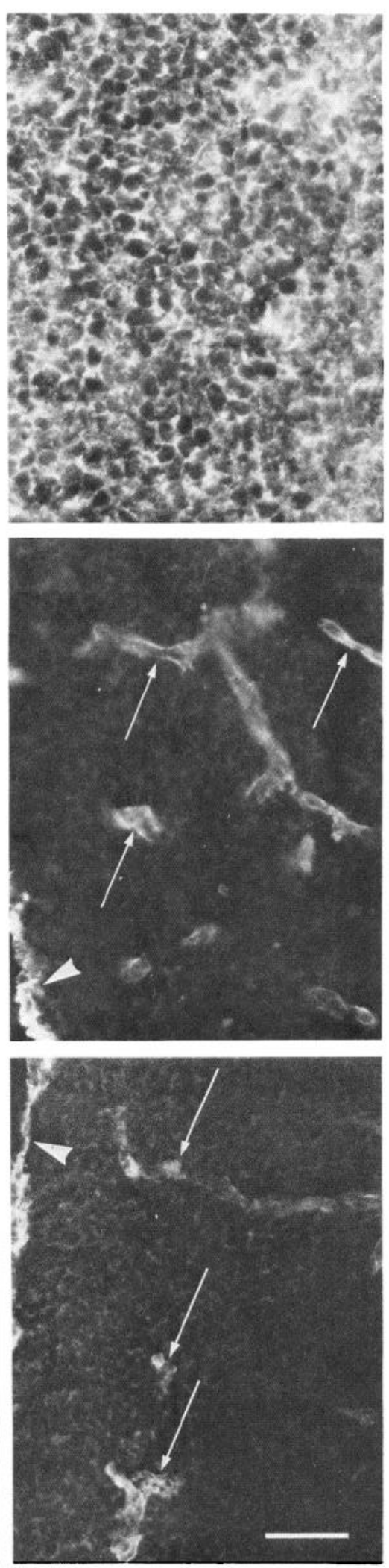

FON
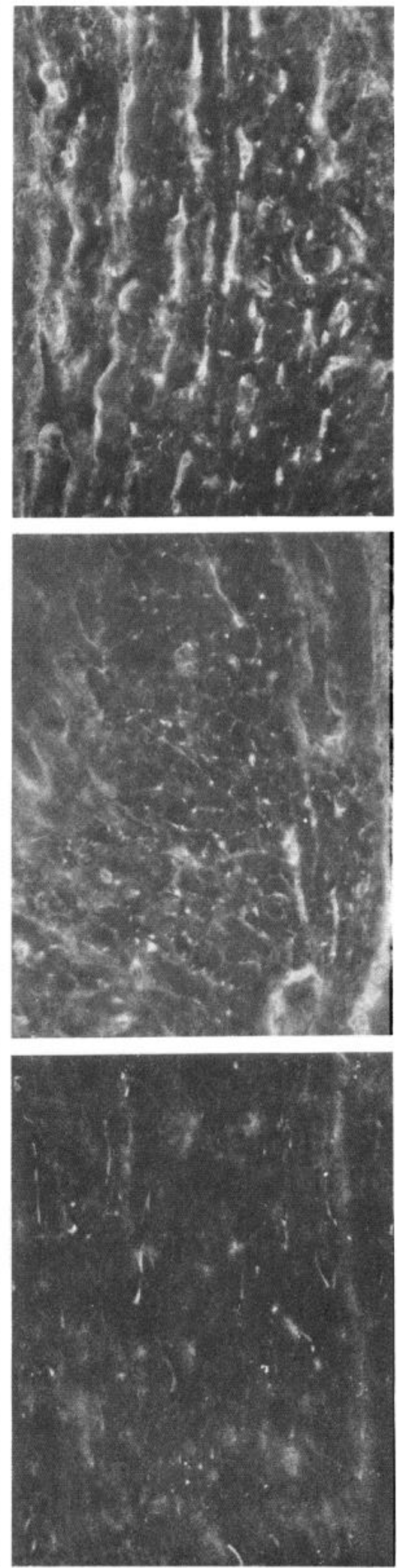

Figure 11. Immunocytochemistry of laminin $(L A M)$, fibronectin $(F N)$, and heparan sulfate proteoglycan $(H S)$ in longitudinal sections of adult rat sciatic nerve $(S N)$, rat embryonic (14.5 d) spinal cord $(E S C)$, and mature goldfish optic nerve $(F O N)$. LAM, FN, and HS immunoreactivities are all found in sciatic nerve sections, located in the endoneurial extracellular matrix, fluorescence for FN being somewhat stronger in the perineurium (arrow in FN/SN). Strong immunoreactivity for LAM is also found around most cells of the rat embryonic spinal cord (LAM/ESC) and is present as well in the fish optic nerve (LAM/FON), although its cellular localization is more diffuse. In contrast, FN and HS immunoreactivities in embryonic spinal cord (ESC) are restricted to connective tissue (arrowheads) and blood vessels (arrows), as in the adult (not shown). The fish optic nerve (FON) displays only low levels of FN and HS immunoreactivities, in punctate and fibrillar forms. Scale bar in HS/ESC represents $50 \mu \mathrm{m}$ and applies to all micrographs. 
glycan in the ECM of sciatic nerve inhibits nerve fiber growth on sciatic nerve sections. If this antibody inhibits the activity of a single species of molecule, then there is only one positive effector. However, the mode of action of this antibody appears to involve binding to a heparan sulfate proteoglycan-laminin complex in an as yet undescribed manner. Thus, the number and identity of the positive effector(s) and of the negative one(s), if any, remain to be detailcd.

In keeping with observations made by others (Schachner et al., 1978; Paetau, 1980; Cornbrooks et al., 1983; Laurie et al., 1983), laminin, fibronectin, and heparan sulfate proteoglycan can be immunocytochemically identified in the endoneurium of sciatic nerve. These antigens are much less prominent in adult rat CNS. The distribution of fibronectin and heparan sulfate proteoglycan was essentially the same in the embryonic and adult rat tissues, i.e., absent throughout the brain and restricted to vascular and connective tissue basement membranes. In contrast, a high level of laminin immunoreactivity is found around most cells of the spinal cord of rat embryos (14-16 d). Similarly, laminin is readily visualized in goldfish optic nerve (Hopkins et al., 1985; Liesi, 1985b), while fibronectin and heparan sulfate proteoglycan are present in low but detectable levels. Thus, laminin is demonstrable in all of the tissue substrata that support nerve fiber growth.

The laminin distribution we found in the embryonic rat spinal cord and goldfish optic nerve is very similar to that reported by Liesi for embryonic rat brain (1985a) and goldfish optic nerve (1985b). Others have failed to detect laminin immunohistochemically in embryonic rat brain and spinal cord (Bignami et al., 1984) or in the developing chick CNS (Rogers et al., 1986). These discrepancies may result from differences in fixation procedures, antisera, embryonic age, etc. Alternatively, our antisera to laminin may be cross-reacting with other molecules. ECM components are well known to copurify (Bayne et al., 1984), which presents difficulties in producing monospecific antisera to them. It is noteworthy that we have found the same distribution of laminin with 2 different antisera, one commercial (Bethesda Research Labs) and one produced by ourselves. The latter is highly specific for laminin and does not cross-react with fibronectin or collagen (S. Carbonetto, unpublished observations). We are presently producing monoclonal antibodies to laminin, which should help considerably in resolving this important point concerning the distribution of laminin in embryonic CNS.

Our studies have focused on the adhesive proteins of the ECM (laminin, fibronectin, heparan sulfate proteoglycan). Another class of adhesive molecules (cell adhesion molecules, or CAMs) also affects nerve fiber growth (Rutishauser et al., 1978) and could be involved in the growth of nerve fibers on our nerve tissue substrata. However, we see little growth on CNS tissue, which, like PNS, has NCAM and NgCAM (reviewed by Edelman, 1984). To a first approximation, this suggests that CAMs are not involved in the differential growth of fibers on these tissues.

The amounts, forms, and distribution of adhesive proteins in the ECM of the nervous system are important determinants of a neuronal microenvironment's ability to support nerve fiber growth, as shown by a number of culture experiments and by correlative studies in vivo (discussed above). However, it is likely that regeneration of nerve fibers in vivo is multifactorial. Consider that some CNS neurons fail to regenerate into PNS grafts in adult rats (Aguayo, 1985). The presence in PNS, but not in CNS, sections of ECM molecules mediating neuronsubstratum adhesion suggests to us that the failure of nerve fiber growth within the adult CNS microenvironment results, in part, from its unsuitability as an adhesive substratum for nerve fiber growth in vivo.

\section{References}

Aguayo, A. J. (1985) Axonal regeneration from injured neurons in the adult mammalian central nervous system. In Synaptic Plasticity, C. W. Cotman, ed., Guilford, New York.

Aguayo, A. J., R. Dickson, J. Trecarten, and M. Attiwell (1978) Ensheathment and myelination of regenerating PNS fibers by transplanted optic nerve glia. Neurosci. Lett. 9: 97-104.

Aguayo, A. J., S. David, P. Richardson, and G. M. Bray (1979) Axonal elongation in peripheral and central nervous system transplants. Adv. Cell Neurobiol. 3: 215-234.

Akers, R. M., D. F. Mosher, and J. E. Lilien (1981) Promotion of retinal neurite outgrowth by substratum-bound fibronectin. Dev. Biol. 86: $179-188$

Barde, Y. A., D. Edgar, and H. Thoenen (1982) Purification of a new neurotrophic factor from mammalian brain. EMBO J. 1: 549-553.

Baron van Evercooren, A., H. K. Kleinman, S. Ohno, P. Marangos, J. P. Schwartz, and M. E. Dubois-Dalq (1982) Nerve growth factor, laminin and fibronectin promote neurite growth in human fetal sensory ganglion cultures. J. Neurosci. Res. 8: 179-194.

Bayne, E. K., M. J. Anderson, and D. M. Fambrough (1984) Extracellular matrix organization in developing muscle: Correlation with acetylcholine receptor aggregates. J. Cell Biol. 99: 1486-1501.

Berg, D. K. (1984) New neuronal growth factors. Annu. Rev. Neurosci. 7: $149-170$.

Bignami, A., N. H. Chi, and D. Dahl (1984) First appearance of laminin in peripheral nerve, cerebral blood vessels and skeletal muscle. Int. J. Dev. Neurosci. 2: 367-376.

Bottenstein, J. E., and G. H. Sato (1978) Growth of a rat neuroblastoma cell line in serum-free supplemented medium. Proc. Natl. Acad. Sci. USA 76: 514-517.

Campenot, R. B. (1977) Local control of neurite development by nerve growth factor. Proc. Natl. Acad. Sci. USA 74: 4516-4519.

Carbonetto, S., and D. M. Fambrough (1978) Synthesis, insertion in the plasma membrane and turnover of $\alpha$-bungarotoxin receptors in chick sympathetic neurons. J. Cell Biol. 81: 555-569.

Carbonetto, S., M. M. Gruver, and D. C. Turner (1982) Nerve fiber growth on defined hydrogel substrates. Science 216: 897-899.

Carbonetto, S., M. M. Gruver, and D. C. Turner (1983) Nerve fiber growth in culture on fibronectin, collagen and glycosaminoglycan substrates. J. Neurosci. 3: 2324-2335.

Collins, F. (1978) Induction of neurite outgrowth by a conditioned medium factor bound to culture substratum. Proc. Natl. Acad. Sci. USA 75: 5210-5213.

Cornbrooks, C. J., D. J. Carey, J. A. McDonald, R. Timpl, and R. P. Bunge (1983) In vivo and in vitro observations on laminin production by Schwann cells. Proc. Natl. Acad. Sci. USA 80: 3850-3854.

Culp, L. A., R. Ansbacher, and C. Domen (1980) Adhesion sites of neural tumor cells: Biochemical composition. Biochemistry 19:58995907.

David, S., and A. J. Aguayo (1981) Axonal elongation into peripheral nervous system "bridges" after central nervous system injury. Science 214: 931-933.

Davis, G. E., E. Engvall, and S. Varon (1985) Isolation and characterization of rat schwannoma neurite-promoting factor: Evidence that the factor contains laminin. J. Neurosci. 5: 2662-2671.

DeGeorge, J. J., N. Slepecky, and S. Carbonetto (1985) Concanavalin A stimulates ncuron-substratum adhesion and nerve fiber outgrowth in culture. Dev. Biol. 111: 335-351.

Edelman, G. M. (1984) Modulation of cell adhesion during induction, histogenesis, and perinatal development of the nervous system. Annu. Rev. Neurosci. 7: 339-377.

Edgar, D., R. Timpl, and H. Thoenen (1984) The heparin binding domain of laminin is responsible for its effects on neurite outgrowth and neuronal survival. EMBO J. 3: 1463-1468.

Greene, L. A., and E. M. Shooter (1980) The nerve growth factor: Biochemistry, synthesis and mechanism of action. Annu. Rev. Neurosci, 3: 353-402. 
Greene, L. A., and A. S. Tischler (1976) Establishment of a noradrernergic clonal cell line of rat adrenal pheochromocytoma cells which respond to nerve growth factor. Proc. Natl. Acad. Sci. USA 73: 2424 2428.

Hawrot, E. (1980) Cultured sympathetic neurons: Effects of cell-derived and synthetic substrata on survival and development. Dev. Biol. 74: 136-151.

Helfand, S. L., G. A. Smith, and N. K. Wessells (1976) Survival and development in culture of dissociated parasympathetic neurons from ciliary ganglia. Dev. Biol. 50: 541-547.

Hopkins, J. M., S. Ford-Holevinski, J. P. McCoy, and B. W. Agranoff (1985) Laminin and optic nerve regeneration in the goldfish. J. Neurosci. 5: 3030-3038.

Ide, C., K. Tohyama, R. Yokota, T. Nitatori, and S. Onodera (1983) Schwann cell basal lamina and nerve regeneration. Brain Res. 288; 61-75.

Klebe, R. J., K. L. Bentley, and R. C. Schoen (1981) Adhesive substrates for fibronectin. J. Cell Physiol. 109: 481-488.

Lander, A. D., D. K. Fujii, and L. F. Reichardt (1985) Laminin is associated with the "neurite outgrowth-promoting factors" found in conditioned media. Proc. Natl. Acad. Sci. USA 82: 2183-2187.

Laurie, G. W., C. P. Leblond, and G. R. Martin (1983) Light microscopic immunolocalization of type IV collagen, laminin, heparan sulfate proteoglycan and fibronectin in the basement membranes of a variety of rat organs. Am. J. Anat. 167: 71-82.

Letourneau, P. C. (1975) Cell to substratum adhesion and guidance of axonal elongation. Dev. Biol. 44: 92-101.

Liesi, P. (1985a) Do neurons in the vertebrate CNS migrate on laminin? EMBO J. 4: 1163-1170.

Liesi, P. (1985b) Laminin-immunoreactive glia distinguish regenerative adult CNS systems from non-regenerative ones. EMBO J. 4: 2505-2511.

Ludueña, M. A. (1973) Nerve cell differentiation in vitro. Dev. Biol. 33: 268-289.

Manthorpe, M., E. Engvall, E. Ruoshlahti, F. M. Longo, G. E. Davis, and S. Varon (1983) Laminin promotes neuritic regeneration from cultured peripheral and central neurons. J. Cell Biol. 97: 1882-1890.

Nathaniel, E. J. H., and D. R. Nathaniel (1973) Regeneration of dorsal root fibers into the adult rat spinal cord. Exp. Neurol. 40: 333-350.

Nathaniel, E. J. H., and D. C. Pease (1963) Regenerative changes in rat dorsal roots following Wallerian degeneration. J. Ultrastruct. Res. 9: 533-549.
Paetau, A., K. Mellstrom, A. Vaheri, and M. Haltia (1980) Distribution of a major connective tissue protein, fibronectin, in normal and neoplastic human nervous tissue. Acta Neuropathol. 51: 47-51.

Rauvala, H. (1984) Neurite outgrowth of neuroblastoma cells: Dependence on adhesion-surface, cell-surface interactions. J. Cell Biol. 98: 1010-1016.

Richardson, P. M., U. M. McGuinness, and A. J. Aguayo (1980) Axons from CNS neurones regenerate into PNS grafts. Nature 284: 264 265.

Rogers, S. L., P. C. Letourneau, S. L. Palm, J. McCarthy, and L. T. Furcht (1983) Neurite extension by peripheral and central nervous system neurons in response to substratum-bound fibronectin and laminin. Dev. Biol. 98: 212-220.

Rogers, S. L., K. J. Edson, P. C. Letourneau, and S. C. McLoon (1986) Distribution of laminin in the developing peripheral nervous system of the chick. Dev. Biol. 113: 429-435.

Ruffolo, R. R., G. S. Eisenbarth, J. M. Thompson, and M. Nirenberg (1978) Synapse turnover: A mechanism for acquiring synaptic specificity. Proc. Natl. Acad. Sci. USA 75: 2281-2285.

Rutishauser, U., W. E. Gall, and G. M. Edelman (1978) Adhesion among neural cells of the chick embryo. IV. Role of the cell surface molecule CAM in the formation of neurite bundles in cultures of spinal ganglia. J. Cell Biol. 79: 382-393.

Sandrock, A. W., and W. D. Matthew (1985) A bioassay for the analysis of neurite promoting factors in the extracellular matrix of rat peripheral nerve. Soc. Neurosci. Abstr. 11: 592.

Schachner, M., G. Schoonmaker, and R. O. Hynes (1978) Cellular and subcellular localization of LETS protein in the nervous system. Brain Res. 158: 149-158.

Schwab, M., and H. Thoenen (1985) Dissociated neurons regenerate into sciatic but not optic nerve explants in culture irrespective of neurotrophic factors. J. Neurosci. 5: 2415-2423.

Smalheiser, N. R., S. M. Crain, and L. M. Reid (1984) Laminin as a substrate for retinal axons in vitro. Dev. Brain Res. 12: 136-140.

Steel, R. G. D., and J. H. Torrie (1980) Principles and Procedures of Statistics: A Biometrical Approach, pp. 173-177, McGraw-Hill, New York.

Wood, P. M. (1976) Separation of functional Schwann cells and neurons from normal peripheral nerve tissue. Brain Res. 115: 361-375. 